\title{
Surface Modification of Tungsten Tailings
}

\author{
Youyu $\mathrm{Li}^{1,2, a}$,Ying Zhang ${ }^{1,2, \mathrm{~b}^{*}}$ \\ 1-State Key Laboratory of Complex Nonferrous Metal Resources Clean Utilization, Kunming \\ 650093,China; \\ 2-Faculty of Land Rescource Engineering,Kunming University of Science and Technology,Kunming \\ 650093,Yunnan,China \\ a1065891183@qq.com, bhyingcsu@163.com
}

Corresponding Author:Ying Zhang,zhyingcsu@163.com

Keywords: tungsten tailings, surface modification, aluminate

Abstract: The tungsten tailings include amount of $\mathrm{SiO}_{2}, \mathrm{CaO}, \mathrm{Al}_{2} \mathrm{O}_{3}$. Tungsten tailings were modified by aluminate as modifier.The activation index of tungsten tailings powder was $64.5 \%$, when aluminate dosage was $1.2 \%$.FT-IR results showed that there were two new peaks which were vibration characteristic absorption peak of $\mathrm{C}-\mathrm{H}$ from aluminate.Chemisorption existed between ttungsten tailings and aluminate.Modified tungsten tailings can be used as filler of rubber high polymer.

\section{Introduction}

Because of the increasingly high demand for environmental protection, further processing of tungsten tailings have been put on the agenda.The main components of tungsten tailings are $\mathrm{SiO}_{2}, \mathrm{CaO}, \mathrm{Al}_{2} \mathrm{O}_{3}$ and so on.Tungsten tailings can be used as cement admixture preparation[1],concrete admixture,plastic packing,glass-ceramics[2], environ -mental-friendly cement mineralizer[3],mineral polymeric materials[4], and so on.

This paper studies the surface-modified of tungsten tailings which can be used for filler of Rubber high polymer.

\section{Experimental materials}

The tungsten tailings is from Xintianling tungsten ore,Hunan province of China.The chemical analysis and size compositions were shown in Table 1 and Table 2 respectively.

Table 1 Chemical analysis of tungsten tailings, $\%$

\begin{tabular}{cccccccc}
\hline Item & $\mathrm{Al}_{2} \mathrm{O}_{3}$ & $\mathrm{CaO}$ & $\mathrm{MgO}$ & $\mathrm{SiO}_{2}$ & $\mathrm{Fe}_{2} \mathrm{O}_{3}$ & $\mathrm{~F}$ & $\mathrm{Ig}$ \\
\hline Grade & 8.70 & 28.02 & 1.12 & 36.52 & 11.71 & 0.80 & 2.30 \\
\hline
\end{tabular}

Table 2 The size compositions of tungsten tailings

\begin{tabular}{lccccc}
\hline Size $(\mu \mathrm{m})$ & +350 & $-350 \sim$ & $-150 \sim+74$ & $-74 \sim+38$ & -38 \\
\hline Content $(\%)$ & 0.16 & 1.90 & 23.20 & 62.01 & 12.67 \\
\hline
\end{tabular}


Reagents which were used for surface modification of tungsten tailings included aluminate[5],titanate, silane and ethanol.

\section{Experimental method}

The powder of tungsten tailings is put in a high-speed mixer,heated under stirring for some time so that the powder moisture content is less than 1\%.A certain proportion of the coupling agent are added to the organic auxiliaries.By vigorous stirring coupling agent is dispersed in organic auxiliaries adequately. The mixed reagent is added into high-speed mixer slowly by spray equipment. Increasing the temperature of the curing process, and then the dehydration reaction is accelerated.In order to better dispersion of the modifier and reaction with powder,we should increase the speed of mixer. Stop high-speed mixer operation after the action time,and then unloading and bagging after cooling temperature.Modified flow chart was shown in Fig. 1.

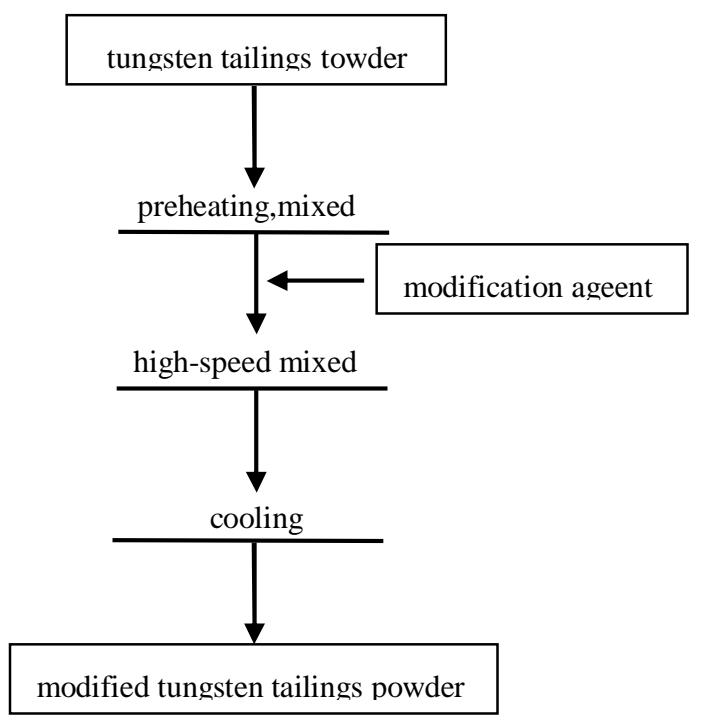

Fig. 1 Modified flow chart of tungsten tailings towder

\section{Results and discussion}

\section{Activation index}

Because the surface hydrophilic and its own weight,tungsten tailings+titanatepowder settles naturally in water. After the surface modification treatment, the surface of powder is from hydrophilic to hydrophobic. When the powder particles are smaller, because of its enormous surface tension and hydrophobic powders float on the water such as film.Modified powder stirs in water or organic matter efficiently,then the solution is stilled and clarified. Activation index which can characterize activation degree of the modified powder is the ratio of weight floats and the total sample.The mathematical expression is that:

$$
\mathrm{H}=\frac{\mathrm{F}}{\mathrm{T}} \times 100
$$

Where $\mathrm{H}$ replace the activation index, $\mathrm{F}$ is weight floats and $\mathrm{T}$ is the total sample.

Activation index value is from 0 to 100 . As the increase of ctivity index, the modification effect is better. 
The tungsten tailings powder were modified by aluminate,titanate and silane respectively. The results were shown in Fig. 2,Fig. 3 and Fig.4.

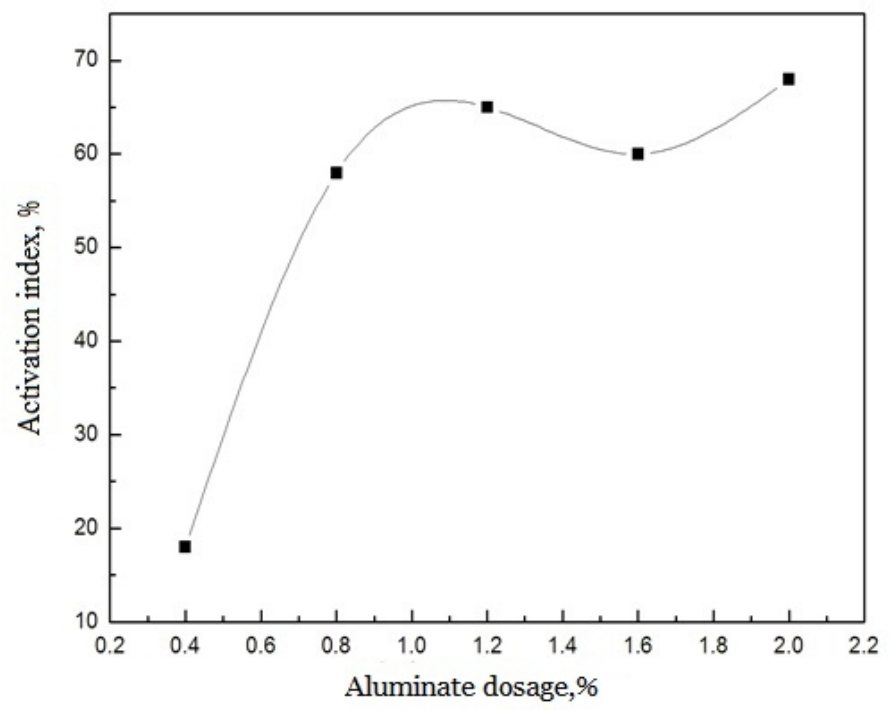

Fig.2 The effect of aluminate dosage on activation index

From the Fig.2, with the increase of aluminate dosage ,the activation index was increased.After the aluminate dosage reached $1.2 \%$, the activation index didn't increase with the increase of aluminate dosage .So the optimum aluminate dosage was $1.2 \%$ and the activation index reached $64.5 \%$.

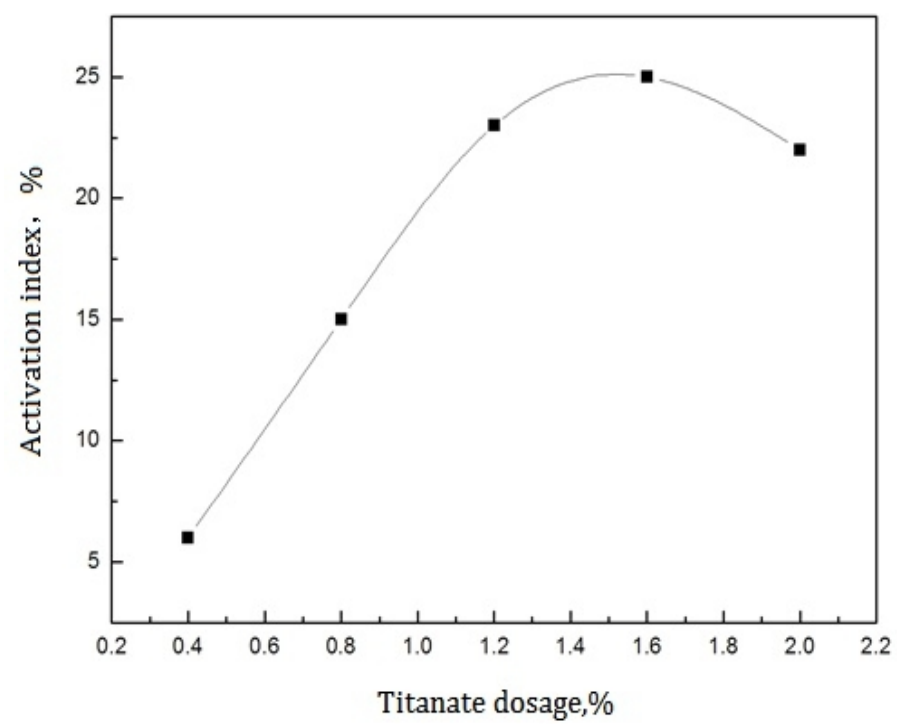

Fig.3 The effect of titanate dosage on activation index

From the Fig.3, with the increase of titanate dosage ,the activation index was increased.After the aluminate dosage reached $1.6 \%$, the activation index was lower. The maximum activation index was $25.1 \%$. 


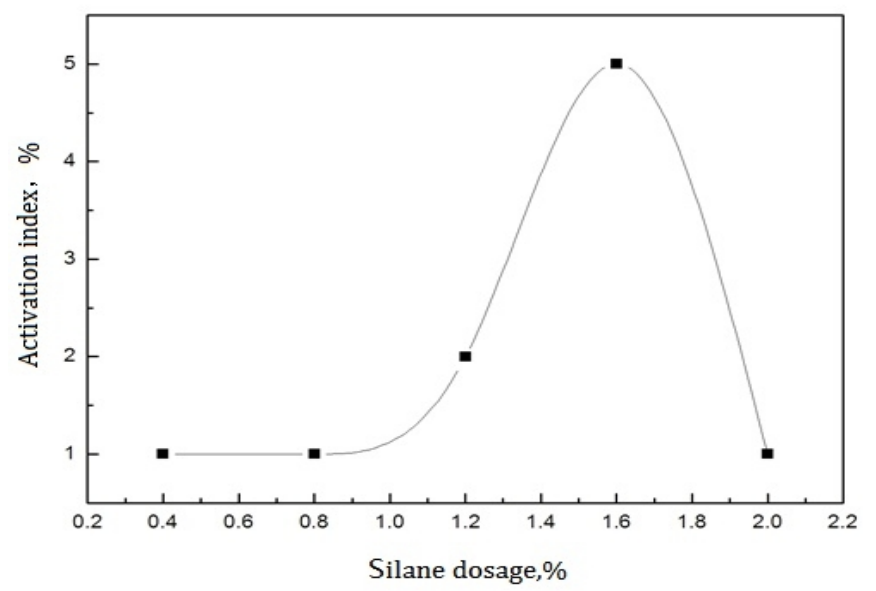

Fig.4 The effect of silane dosage on activation index

From the Fig. 4 , when the silane dosage increased from $0.4 \%$ to $0.8 \%$, the activation index remained unchanged.With the increase of titanate dosage to $1.6 \%$, the activation index was increased,and the maximum was only $4.98 \%$. With the continued increase of titanate dosage,the activation index was reduced to $1 \%$.

Considering Fig.2,Fig.3 and Fig.4 ,aluminate dosage is the best modifier for tungsten tailings powder, the activation index is more than $65 \%$.

\section{Infrared spectrum(FT-IR) analysis}

The surface modifiers which react by chemical bond with he surface of the powder particles are used widely in the application process.Physical adsorption are desorbed easily under vigorous stirring or compression, so that it can effcct compatibility, wettability and dispersion of tungsten tailings powder in the polymer .

The chemically bound state between elements of modified powder can be analyzed through FT-IR.The FT-IR results before and after the tungsten tailings modified are shown in Fig.5.

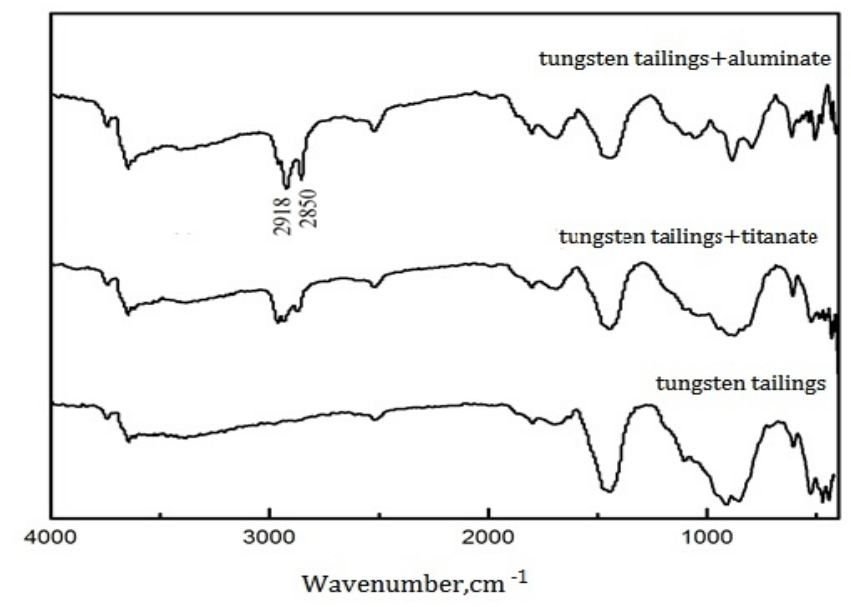

Fig.5 The FT-IR results before and after the tungsten tailings modified

There were two new methyl peak at $2918 \mathrm{~cm}^{-1}$ and $2850 \mathrm{~cm}^{-1}$ after modification.The new peaks were vibration characteristic absorption peak of $\mathrm{C}-\mathrm{H}$ of methylene and methine which were introduced by 
aluminate and titanate.The FT-IR analysis shows that chemical adsorption occured between tungsten tailings with a modifier.

\section{Conclusions}

1) The activation index of tungsten tailings which was modified by aluminate was highest.The modification effect of aluminate was better than titanate and silane. When the aluminate dosage was $1.2 \%$, the activation index reached $64.5 \%$.

2) The reaction between tungsten tailings and aluminate was chemical action.

3) FT-IR results were consistent with the modified experimental results.

\section{Acknowledgements}

The authors would like to express their gratitude for the financial support from the National Natural Science Foundation of Youth Project (51504108).

\section{References}

[1] D.G.Su,S.M.Lin.Application of Wolfram Tailings in Cement Industry.Multipurpose Utilization of Mineral Resources,10(2003),9.50-52.

[2] J.Z.Kuang,S.H.Xiong.The Composition of Tungsten Tailings Glass-ceraics and Its Manufacture.Multipurpose utilization of Mineral resources,3(2003),p.37-39.

[3] D.G. Liu,X.T. Zhou.On the Utilization of Tungsten Tailings as Environmental -friendly Cement Mineralizer.China Tungsten Industry,22(2007),p.31-33.

[4] J.Z. Kuang,F.Shi,T.S.Qiu,J.Z.Chen .Study on influence factors of geopolymerin preparation with tungsten-tailing.Concrete,11(2009),p.71-77.

[5] S.T. Liu, C. Ma, W.D. Cao, J.G. Fang.Influence of aluminate coupling agent on low-temperature rheological performance of asphalt mastic.Construction and Building Materials, 24(2010), p.650-659. 